\title{
Screening of potential hub genes in pulmonary thromboembolism
}

\author{
YAN LU $^{1 *}$, JUN DAI $^{1 *}$, QIYANG LIU ${ }^{1}$, PAN CAI $^{2}$ and JIANGHUA ZHENG ${ }^{1}$ \\ Departments of ${ }^{1}$ Laboratory Medicine and ${ }^{2}$ Orthopedic Surgery, Shanghai University of Medicine \\ and Health Sciences Affiliated Zhoupu Hospital, Shanghai 201318, P.R. China \\ Received November 6, 2020; Accepted February 4, 2021
}

DOI: 10.3892/etm.2021.10940

\begin{abstract}
Pulmonary thromboembolism (PTE) is a fatal clinical syndrome that usually occurs in elderly individuals. The present study aimed to identify functional and key genes involved in the early diagnosis of PTE using bioinformatics analysis. The GSE84738 dataset was retrieved from the Gene Expression Omnibus database. Gene Ontology and Kyoto Encyclopedia of Genes and Genomes pathway enrichment analyses were subsequently performed. In addition, Cytoscape software v.3.7.2 was used to construct a protein-protein interaction (PPI) network. Serum samples from patients with PTE and healthy individuals were collected and the expression levels of Toll-like receptor (TLR)4, TLR2, IL-1 $\beta$, JUN, prostaglandin-endoperoxide synthase 2 (PTGS2), osteopontin (SPP1) and endothelin-1 (ET-1) were analyzed by reverse transcription-quantitative PCR. A total of 160 upregulated and 159 downregulated differentially expressed genes were identified between patients with PTE and healthy individuals. TNF, IL-1 $\beta$, JUN, TLR4, PTGS2, vascular cell adhesion molecule 1, SPP1, ryanodine receptor 2, TLR2 and ET-1 were considered as hub genes, which are defined as the genes with the highest degree of interaction in the enrichment and PPI network analyses. The top 10 common genes with the highest degree in the PPI network and the top 10 genes in modules 1 and 2 were TLR4, TLR2, IL-1 $\beta$, JUN, PTGS2, SPP1 and ET-1. Taken together, the present study suggested that TLR4, TLR2, IL-1 $\beta$ and SPP1 were enriched in patients with PTE, thus providing novel potential biomarkers for the diagnosis of PTE.
\end{abstract}

Correspondence to: Dr Pan Cai, Department of Orthopedic Surgery, Shanghai University of Medicine and Health Sciences Affiliated Zhoupu Hospital, 1500 Zhouyuan Road, Shanghai 201318, P.R. China

E-mail: zp_caip@sumhs.edu.cn

Dr Jianghua Zheng, Department of Laboratory Medicine, Shanghai University of Medicine and Health Sciences Affiliated Zhoupu Hospital, 1500 Zhouyuan Road, Shanghai 201318, P.R. China

E-mail: zhengjianghua1500@126.com

${ }^{*}$ Contributed equally

Key words: pulmonary thromboembolism, biomarker, bioinformatics, Gene Expression Omnibus

\section{Introduction}

Pulmonary thromboembolism (PTE), a fatal condition mainly affecting older individuals, ranks third among the most common types of cardiovascular diseases after coronary heart disease and high blood pressure (1). At present, the prevention and control of PTE is frequently inadequate, particularly in patients with multiple traumas; therefore, the diagnosis of PTE is frequently missed, which in turn leads to a high mortality rate for patients with PTE (2). It is well known that PTE is caused by an endogenous or exogenous embolus blocking the pulmonary artery or its main branches (3). The pneumovascular bed has a great reserve capacity. One of the major functions of the lung is blood filtration, thereby preventing small thrombi from flowing into the systemic circulation (4). Furthermore, lung tissue exerts strong autolysis and dissolving effects on small thrombi. Therefore, in clinical practice, when a small thrombus blocks the pulmonary vascular bed, clinical symptoms frequently do not appear due to the self-dissolving effect of the lung tissue, also known as clinical non-dominant pulmonary embolism. These reports indicate that the early clinical diagnosis of PTE is challenging (5). Biomarkers may not only reveal the pathological process at the molecular level but also have the advantage of accurately assessing low-level and early injury, thereby providing an early warning and, to a large extent, the basis for diagnosis by clinicians (6). The present study aimed to identify functional key genes for the early diagnosis of PTE using bioinformatics analysis.

Bioinformatics is an interdisciplinary field incorporating biology and computer science in order to analyze and synthesize related data (7). Several bioinformatics tools are currently used to screen for potential genes associated with a variety of pathological processes in vivo $(8,9)$. Similarly, microarrays have been used to elucidate underlying pathological mechanisms and identify novel biological markers (10). Recently, microarrays have been utilized by several researchers to reveal key genes associated with thrombotic diseases and several relevant genes have been identified, including Janus kinase 2, stabilin 2 and vitamin K-dependent protein $\mathrm{S}(11,12)$. These differentially expressed genes (DEGs) are assumed to have a potential role in thrombosis by regulating blood coagulation.

In the present study, the microarray data of the GSE84738 dataset were analyzed in order to identify DEGs associated with PTE. In addition, functional enrichment analysis of the 
DEGs was performed. The protein-protein interaction (PPI) network of the DEGs was constructed to screen out the hub genes and the differential expression of certain hub genes was validated in serum samples from patients with PTE and healthy controls.

\section{Materials and methods}

Datasets and DEGs. The microarray dataset GSE84738 containing data from a rabbit model of PTE was downloaded from the Gene Expression Omnibus (GEO) database (https://www.ncbi.nlm.nih.gov/geo/). Subsequently, the data were analyzed with the Morpheus online tool (https://software.broadinstitute.org/morpheus). The expression heatmap was ultimately displayed. The criteria for DEGs were a signal-to-noise ratio of $>1$ or $<-1$. Signal-to-noise ratio is the amount of biological signal relative to the amount of noise (13). Using this method, Mi et al (13) obtained a satisfactory DEGs identification efficiency in the study.

Gene ontology $(G O)$ and kyoto encyclopedia of genes and genomes $(K E G G)$ analyses. The Database for Annotation, Visualization and Integrated Discovery (DAVID; version 6.8; david.ncifcrf.gov/) online tool was utilized to perform GO enrichment and KEGG pathway analyses (14). The results were visualized using the R package 'GOplot' and 'ggplot'.

PPI network construction. The functional interactions among DEGs were analyzed by constructing a PPI network. In brief, DEGs were imported into the Search Tool for the Retrieval of Interacting Genes database (STRING; www.string-db.org) and a combined score of $>0.5$ was set as the cut-off value to indicate significant interactions. The combined score is computed by combining the probabilities from the different evidence channels and corrected for the probability of randomly observing an interaction (15). Furthermore, the PPI network among DEGs was established using the Cytoscape analysis software (version 3.7.2) (16). Significant modules in the PPI network were selected based on the Molecular Complex Detection (MCODE 2.0.0) plugin (https://apps. cytoscape.org/apps/mcode) (17), with scores $\geq 5$ and number of nodes $>5$ in Cytoscape. Ultimately, the DAVID online tool was used to perform functional enrichment analysis of DEGs in the top module and DEGs were ranked based on the degree centrality, calculated using the CentiScaPe 2.2 plugin of Cytoscape. The node degree represents the association degree of one node with all the other nodes in the network. Closeness centrality is a measure of how close a node is to other nodes in the network. Betweenness centrality refers to the number of times a node acts as the shortest bridge between two other nodes.

Patients and blood collection. Peripheral blood samples were collected from 10 patients with PTE and 10 healthy controls admitted to the Shanghai University of Medicine and Health Sciences Affiliated Zhoupu Hospital (Shanghai, China) between June 2019 and April 2020, and the mRNA expression levels of DEGs were determined by reverse transcription-quantitative PCR (RT-qPCR). Patients with PTE included in the present study did not exhibit any other complications following fracture surgery. Blood samples were collected immediately after PTE diagnosis.

RT-qPCR analysis. Total RNA was isolated from serum samples using TRIzol ${ }^{\circledR}$ reagent (Invitrogen; Thermo Fisher Scientific, Inc.). Subsequently, the purified RNA was reverse-transcribed into complementary DNA using the ReverTra Ace ${ }^{\circledR}$ qPCR RT Master mix according to the manufacture's protocol (Toyobo Life Science). The RT reaction was performed at $42^{\circ} \mathrm{C}$ for $15 \mathrm{~min}$, followed by $5 \mathrm{~min}$ at $98^{\circ} \mathrm{C}$. qPCR was performed using a QuantiTect SYBR-Green PCR kit (Qiagen GmbH) on an ABI 7300 Real-Time PCR System (Applied Biosystems; Thermo Fisher Scientific, Inc.). Thermocycling conditions: Initial denaturation for $1 \mathrm{~min}$ at $94^{\circ} \mathrm{C}$, followed by 35 cycles of $1 \mathrm{~min}$ at $60^{\circ} \mathrm{C}, 2 \mathrm{~min}$ at $72^{\circ} \mathrm{C}$, 6 min at $72^{\circ} \mathrm{C}$, holding at $4^{\circ} \mathrm{C}$. The relative mRNA expression levels were normalized to those of the internal control ( $\beta$-actin) and were quantified based on the $2^{-\Delta \Delta C q}$ method (18). The primer sequences used were as follows: Toll-like receptor 4 (TLR4) forward, 5'-AGGATGAGGACTGGGTAA GGA-3' and reverse, 5'-CTGGATGAAGTGCTGGGACA-3'; TLR2 forward, 5'-TTATCCAGCACACGAATACACAG-3' and reverse, 5'-AGGCATCTGGTAGAGTCATCAA-3'; IL-1 $\beta$ forward, 5'-ACAGATGAAGTGCTCCTTCCA-3' and reverse, 5'-GTCGGAGATTCGTAGCTGGAT-3'; osteopontin (SPP1) forward, 5'-GCAUCUUCUGAGGUCAAUUTT-3' and reverse, 5'-AAUUGACCUCAGAAGAUGCTT-3'; JUN forward, 5'-TCCAAGTGCCGAAAAAGGAAG-3' and reverse, 5'-CGA GTTCTGAGCTTTCAAGGT-3'; prostaglandin-endoperoxide synthase 2 (PTGS2) forward, 5'-CTGGCGCTCAGCCAT ACAG-3' and reverse 5'-CGCACTTATACTGGTCAAAT CCC-3'; endothelin-1 (ET-1) forward, 5'-AGAGTGTGTCTA CTTCTGCCA 3 ' and reverse, 5'-CTTCCAAGTCCATACGGA ACAA-3'; $\beta$-actin forward, 5'-GTGGGGCGCCCCAGGCA CCA-3' and reverse, 5'-GCTCGGCCGTGGTGGTGAAG-3'.

Statistical analysis. All data were analyzed using GraphPad Prism 8.0 software (GraphPad Software, Inc.). Values are expressed as the mean \pm standard deviation. Student's t-test or one-way ANOVA followed by Tukey's post hoc test were used to compare the differences between two groups and $>$ two groups, respectively. Fisher's exact test was used to assess significance in the male/female ratio between groups. $\mathrm{P}<0.05$ was considered to indicate a statistically significant difference.

\section{Results}

Identification of DEGs. The expression profile of GSE84738 was downregulated from the GEO database and comprised 4 normal and 4 PTE samples. The blood samples had been collected at seven days following the establishment of the PTE model. The data were analyzed using the Morpheus online tool. A total of 160 upregulated and 159 downregulated DEGs were identified between healthy and PTE cases. The top 30 upregulated and downregulated genes are presented in Fig. 1.

GO and KEGG enrichment analysis. GO and KEGG enrichment analyses were performed using the DAVID online software. In the GO category biological process, 


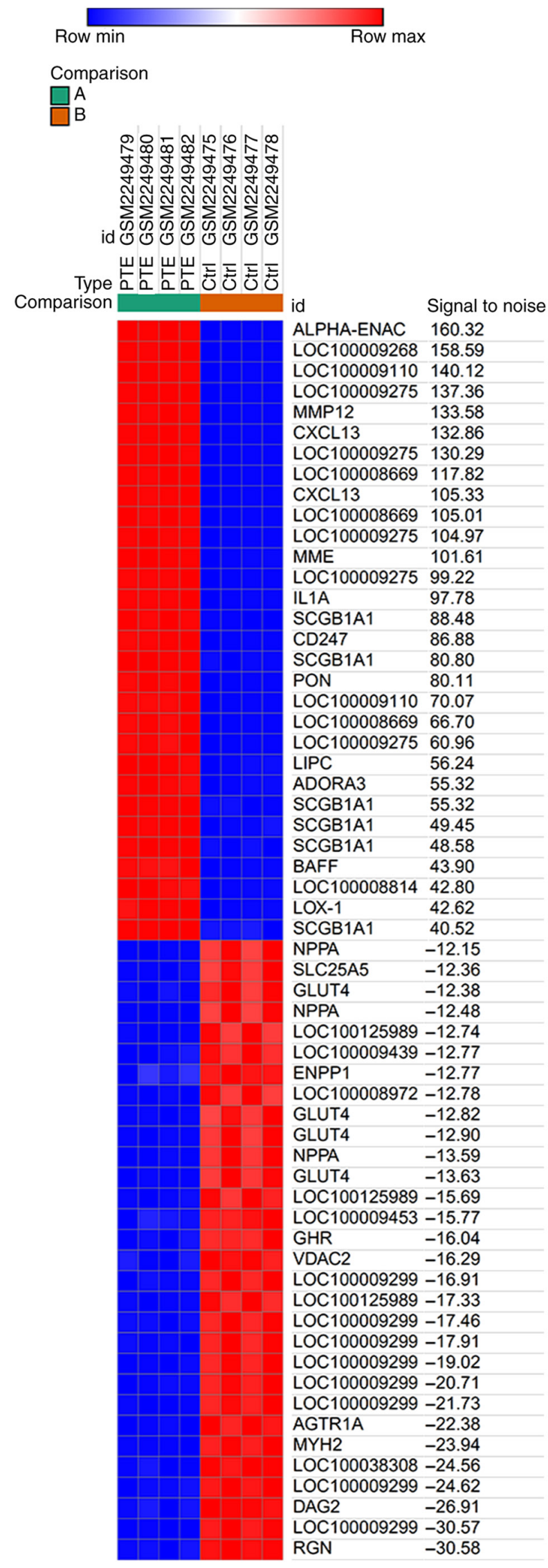

Figure 1. Heat map of the top DEGs in the GSE84738 dataset. Here, 60 upregulated and 60 downregulated DEGs are presented. In total, 160 upregulated and 159 downregulated DEGs were identified. Upregulated genes are presented in red and downregulated genes in blue. DEGs, differentially expressed genes; Ctrl, control; PTE, pulmonary thromboembolism; min, minimum; max, maximum. upregulated DEGs in PTE were significantly enriched in the terms 'immune response', 'defense response', 'inflammatory response', 'positive regulation of immune system process' and 'response to external stimulus', while the downregulated DEGs were enriched in 'ion transport', 'muscle system process', 'metal ion transport', 'muscle contraction' and 'cation transport' (Table I). In the GO category cellular component, upregulated DEGs in PTE were mainly enriched in the terms 'plasma lipoprotein particle', 'lipoprotein particle', 'protein-lipid complex', 'extracellular space' and 'extracellular region' and the downregulated DEGs in 'sarcolemma', 'cation channel complex', 'ion channel complex', 'glycoprotein complex' and 'dystrophin-associated glycoprotein complex' (Table II). Furthermore, in the GO category molecular function, upregulated DEGs in PTE were significantly enriched in the terms 'pattern recognition receptor activity', 'signaling pattern recognition receptor activity', 'low-density lipoprotein particle binding', 'lipopolysaccharide binding' and 'lipoprotein particle binding', while the downregulated ones were mainly enriched in 'voltage-gated ion channel activity', 'voltage-gated channel activity', 'substrate-specific channel activity', 'structural constituent of eye lens' and 'channel activity' (Table III). The top 5 KEGG pathways enriched by the upregulated DEGs in PTE were the 'phagosome pathway', 'rheumatoid arthritis pathway', 'leishmaniasis pathway', 'hematopoietic cell lineage pathway' and 'Chagas disease pathway'. The top 5 KEGG pathways for the downregulated DEGs were 'calcium signaling pathway', 'neuroactive ligand-receptor interaction pathway', 'arrhythmogenic right ventricular cardiomyopathy pathway', 'hypertrophic cardiomyopathy pathway' and 'hypoxia-inducible factor-1 signaling pathway’ (Table IV and Fig. 2).

PPI network analysis. A PPI network was constructed using STRING (Fig. 3). The top 10 genes with the highest node degrees were determined using the Cytoscape software and these genes were TNF, IL-1 $\beta$, JUN, TLR4, PTGS2, vascular cell adhesion molecule 1, SPP1, ryanodine receptor 2, TLR2 and ET-1 (Table V). The scores of closeness centrality, degree centrality and betweenness centrality are presented in Fig. 4. In the PPI network, a total of 5 modules with an MCODE score $\geq 5$ and number of nodes $>5$ were obtained (Table VI).

GO and KEGG functional enrichment analyses. The genes involved in the top two modules were further subjected to GO and KEGG enrichment analysis. GO and KEGG pathway analyses are two important bioinformatics tools that systematically provide insight into how DEGs, including hub genes, exert their biological functions. Therefore, the functions of DEGs in PTE may be predicted using those GO and KEGG pathway analyses. The genes in top module 1 were significantly enriched in the GO terms 'macromolecule localization', 'response to organic substance', 'positive regulation of immune system process', 'plasma lipoprotein particle', 'lipoprotein particle', 'protein-lipid complex', 'low-density lipoprotein particle binding', 'lipoprotein particle binding' and 'protein-lipid complex binding'. In the KEGG pathway analysis, the terms 'rheumatoid arthritis', 'malaria' and 'inflammatory bowel disease' were significantly enriched (Table VII). The genes in top module 2 were mainly enriched in the GO terms 'leukocyte activation', 'immune response', 
Table I. GO analysis of the upregulated and downregulated differentially expressed genes in the category biological process.

A, Upregulated

\begin{tabular}{llrr}
\hline Term & \multicolumn{1}{c}{ Function } & Count & P-value \\
\hline GO:0006955 & Immune response & 32 & $1.9 \times 10^{-11}$ \\
GO:0006952 & Defense response & 33 & $3.5 \times 10^{-11}$ \\
GO:0006954 & Inflammatory response & 20 & $1.0 \times 10^{-9}$ \\
GO:0002684 & Positive regulation of immune system process & 24 & $5.1 \times 10^{-9}$ \\
GO:0009605 & Response to external stimulus & 37 & $3.5 \times 10^{-8}$ \\
\hline
\end{tabular}

B, Downregulated

\begin{tabular}{|c|c|c|c|}
\hline Term & Function & Count & P-value \\
\hline GO:0006811 & Ion transport & 23 & $2.1 \times 10^{-7}$ \\
\hline GO:0003012 & Muscle system process & 13 & $1.1 \times 10^{-6}$ \\
\hline GO:0030001 & Metal ion transport & 17 & $1.2 \times 10^{-6}$ \\
\hline GO:0006936 & Muscle contraction & 11 & $8.4 \times 10^{-6}$ \\
\hline GO:0006812 & Cation transport & 17 & $8.6 \times 10^{-6}$ \\
\hline
\end{tabular}

GO, Gene Ontology.

Table II. GO analysis of the upregulated and downregulated differentially expressed genes in the category cellular component.

A, Upregulated

\begin{tabular}{llrr}
\hline Term & \multicolumn{1}{c}{ Function } & Count & P-value \\
\hline GO:0034358 & Plasma lipoprotein particle & 8 & $4.1 \times 10^{-8}$ \\
GO: 1990777 & Lipoprotein particle & 8 & $4.1 \times 10^{-8}$ \\
GO:0032994 & Protein-lipid complex & 8 & $7.1 \times 10^{-8}$ \\
GO:0005615 & Extracellular space & 28 & $5.1 \times 10^{-7}$ \\
GO:0005576 & Extracellular region & 58 & $3.2 \times 10^{-5}$ \\
\hline
\end{tabular}

B, Downregulated

\begin{tabular}{llrr}
\hline Term & \multicolumn{1}{c}{ Function } & Count & P-value \\
\hline GO:0042383 & Sarcolemma & 8 & $1.4 \times 10^{-5}$ \\
GO:0034703 & Cation channel complex & 9 & $8.0 \times 10^{-5}$ \\
GO:0034702 & Ion channel complex & 10 & $2.0 \times 10^{-4}$ \\
GO:0090665 & Glycoprotein complex & 4 & $3.1 \times 10^{-4}$ \\
GO:0016010 & Dystrophin-associated glycoprotein complex & 4 & $3.1 \times 10^{-4}$ \\
\hline
\end{tabular}

GO, Gene Ontology.

'cell activation', 'myosin complex', 'external side of plasma membrane', 'myofibril, motor activity', 'calcium ion binding' and 'structural constituent of muscle' and in the KEGG pathway terms 'TLR signaling pathway' and 'rheumatoid arthritis' (Table VIII). The enrichment analysis results for modules 1 and 2 are presented in Fig. 5. The common hub genes in the PPI network and those in modules 1 and 2 were
TLR4, TLR2, IL-1 $\beta$, JUN, PTGS2, SPP1 and ET-1. Boxplots indicating the expression levels are presented in Fig. 6.

Validation of differential expression of TLR4, TLR2, IL-1 $\beta$ and SPP1 in patients with PTE. Since the microarray dataset GSE84738 included data from a rabbit model of PTE, it was uncertain whether similar results were able to be obtained 
Table III. GO analysis of the upregulated and downregulated differentially expressed genes in the category molecular function.

A, Upregulated

\begin{tabular}{llrr}
\hline Term & \multicolumn{1}{c}{ Function } & Count & P-value \\
\hline GO:0038187 & Pattern recognition receptor activity & 5 & $3.6 \times 10^{-6}$ \\
GO:0008329 & Signaling pattern recognition receptor activity & 5 & $3.6 \times 10^{-6}$ \\
GO:0030169 & Low-density lipoprotein particle binding & 5 & $8.4 \times 10^{-6}$ \\
GO:0001530 & Lipopolysaccharide binding & 5 & $1.7 \times 10^{-5}$ \\
GO:0071813 & Lipoprotein particle binding & 5 & $3.0 \times 10^{-5}$ \\
\hline
\end{tabular}

B, Downregulated

\begin{tabular}{llrr}
\hline Term & \multicolumn{1}{c}{ Function } & Count & P-value \\
\hline GO:0005244 & Voltage-gated ion channel activity & 15 & $2.7 \times 10^{-10}$ \\
GO:0022832 & Voltage-gated channel activity & 15 & $2.7 \times 10^{-10}$ \\
GO:0022838 & Substrate-specific channel activity & 19 & $1.5 \times 10^{-8}$ \\
GO:0005212 & Structural constituent of eye lens & 7 & $2.4 \times 10^{-8}$ \\
GO:0015267 & Channel activity & 19 & $2.4 \times 10^{-8}$ \\
\hline
\end{tabular}

GO, Gene Ontology.

Table IV. Kyoto Encyclopedia of Genes and Genomes pathway analysis of the upregulated and downregulated differentially expressed genes. The top five terms were selected based on the P-value when more than five enriched terms were identified in each category.

A, Upregulated

\begin{tabular}{llrr}
\hline Term & \multicolumn{1}{c}{ Description } & Count & P-value \\
\hline ocu04145 & Phagosome pathway & 14 & $9.1 \times 10^{-8}$ \\
ocu05323 & Rheumatoid arthritis pathway & 11 & $3.6 \times 10^{-7}$ \\
ocu05140 & Leishmaniasis pathway & 10 & $6.3 \times 10^{-7}$ \\
ocu04640 & Hematopoietic cell lineage pathway & 10 & $7.9 \times 10^{-7}$ \\
ocu05142 & Chagas disease pathway & $9.5 \times 10^{-5}$
\end{tabular}

Downregulated

\begin{tabular}{llrr}
\hline Term & \multicolumn{1}{c}{ Description } & Count \\
\hline ocu04020 & Calcium signaling pathway & 11 & $1.8 \times 10^{-5}$ \\
ocu04080 & Neuroactive ligand-receptor interaction pathway & 11 & $4.7 \times 10^{-4}$ \\
ocu05412 & Arrhythmogenic right ventricular cardiomyopathy pathway & 6 & $7.9 \times 10^{-4}$ \\
ocu05410 & Hypertrophic cardiomyopathy pathway & 6 & $2.3 \times 10^{-3}$ \\
ocu04066 & HIF-1 signaling pathway & $6.1 \times 10^{-3}$ \\
\hline
\end{tabular}

HIF, hypoxia-inducible factor.

from human subjects with PTE. Therefore, the mRNA expression levels of the top seven DEGs were determined in patients with PTE and healthy controls using RT-qPCR analysis. There are no significant differences between the two groups in terms of age, sex distribution and BMI (Table SI). As presented in
Fig. 7, the mRNA levels of TLR4, TLR2, IL-1 $\beta$ and SPP1 were significantly upregulated in patients with PTE compared with those in healthy controls. These results were consistent with those obtained by the bioinformatics analysis. The schema of the study is shown in Fig. 8. 
Table V. Top 10 genes based on degree centrality.

\begin{tabular}{lccr}
\hline Gene ID & Degree & Betweenness & Closeness \\
\hline TNF & 66 & 19868 & 0.00114 \\
IL1B & 40 & 6314 & 0.00108 \\
JUN & 40 & 12052 & 0.00108 \\
TLR4 & 37 & 7908 & 0.00105 \\
PTGS2 & 35 & 4373 & 0.00102 \\
VCAM-1 & 33 & 3006 & 0.00099 \\
SPP1 & 29 & 5227 & 0.00098 \\
RYR-2 & 28 & 5346 & 0.00088 \\
TLR2 & 27 & 1916 & 0.00093 \\
ET-1 & 27 & 6190 & 0.00101 \\
\hline
\end{tabular}

VCAM-1, vascular cell adhesion molecule 1; TLR4, Toll-like receptor 4; TLR2, Toll-like receptor 2; SPP1, Secreted Phosphoprotein 1; RYR-2, Ryanodine Receptor 2; PTGS2, Prostaglandin-Endoperoxide Synthase 2; ET-1, ETS Proto-Oncogene 1, Transcription Factor, IL, interleukin.

Table VI. Scores obtained from five modules from the protein-protein interaction network, which satisfied the criteria of Molecular Complex Detection score $\geq 5$ and number of nodes $>5$.

\begin{tabular}{lccr}
\hline Cluster & Score & Nodes & Edges \\
\hline 1 & 7.333 & 19 & 66 \\
2 & 6.897 & 30 & 100 \\
3 & 5.667 & 7 & 17 \\
4 & 5.600 & 6 & 14 \\
5 & 5.429 & 8 & 19 \\
\hline
\end{tabular}
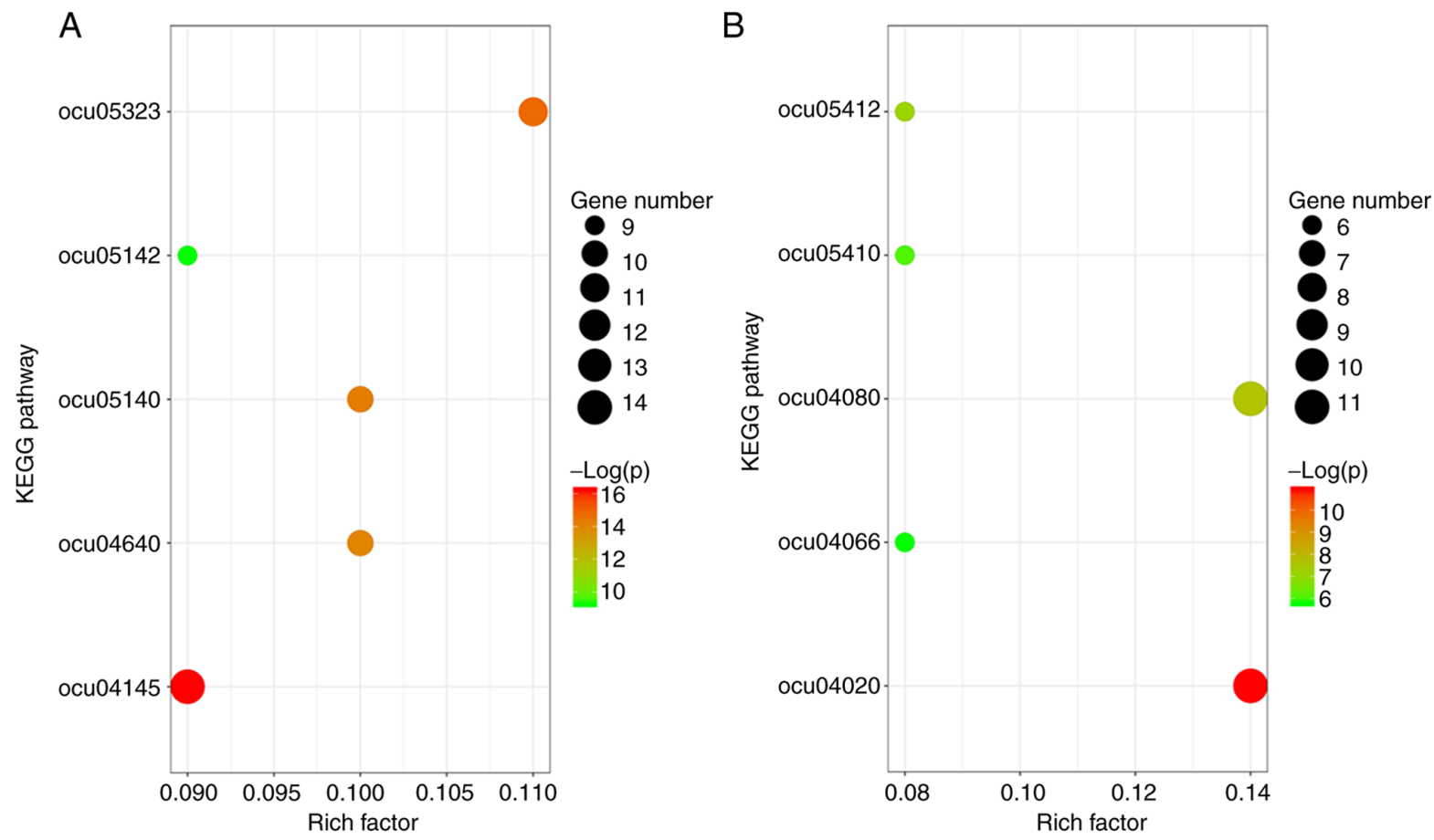

Figure 2. KEGG pathway enrichment analysis results. (A) KEGG pathway enrichment analysis results of the upregulated genes. Pathways: Ocu04145, phagosome pathway; ocu05323, rheumatoid arthritis pathway; ocu05140, leishmaniasis pathway; ocu04640, hematopoietic cell lineage pathway; ocu05142, Chagas disease pathway. (B) KEGG pathway enrichment analysis results of the downregulated genes. Pathways: ocu04020, calcium signaling pathway; ocu04080, neuroactive ligand-receptor interaction pathway; ocu05412, arrhythmogenic right ventricular cardiomyopathy pathway; ocu05410, hypertrophic cardiomyopathy pathway; ocu04066, hypoxia-inducible factor-1 signaling pathway. KEGG, Kyoto Encyclopedia of Genes and Genomes. 


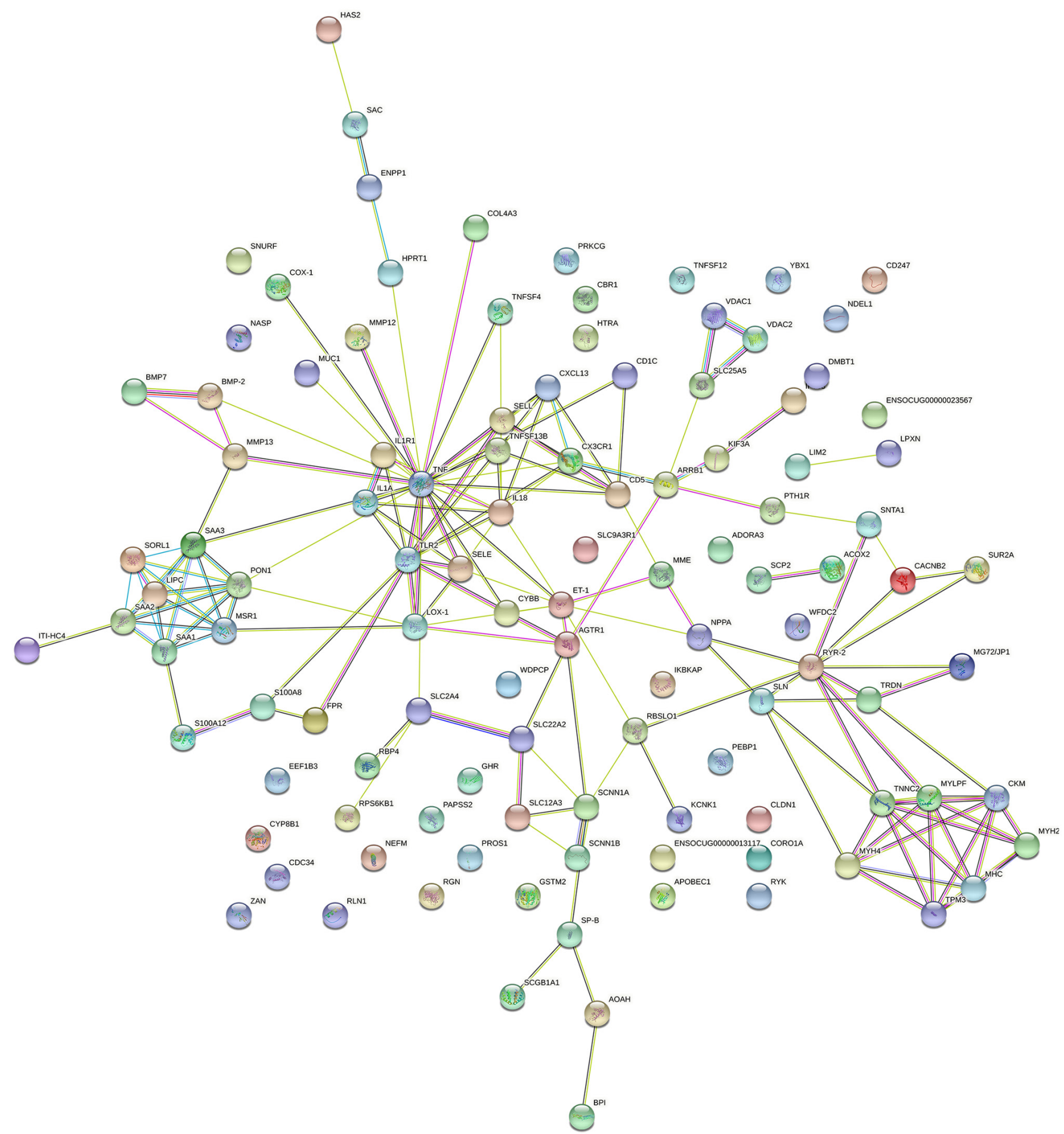

Figure 3. Protein-protein interaction network. This network was constructed using the Search Tool for the Retrieval of Interacting Genes online tool with default settings. Colored nodes represent query proteins and first shell of interactors. Thickness of each edge represent the level of confidence.

\section{Discussion}

In the present study, microarray data from four model rabbits with PTE and four control rabbits were compared and 319 DEGs, including 160 upregulated and 159 downregulated genes, were identified. Furthermore, a PPI network was constructed to reveal the functional interactions between DEGs and the seven most common hub genes between the top 10 genes in the PPI network and the top 10 genes in modules 1 and 2 were screened out using the plugin software
MCODE of the Cytoscape. These genes were IL-1 $\beta$, JUN, TLR4, PTGS2, SPP1, TLR2 and ET-1.

PTE is a fatal clinical syndrome, which is characterized by breathing difficulty, chest pain or discomfort, faster than normal or irregular heartbeat, hemoptysis, low blood pressure, light-headedness or fainting (19). Studies have recently focused on the underlying mechanisms of PTE and several functional genes have been identified as key factors in the development of PTE. For instance, Liu et al (20) reported that the upregulation of stress-associated endoplasmic 
Table VII. Functional and pathway enrichment analysis of the top genes in module 1 . The top three terms were selected based on the P-value when more than three enriched terms were identified in each category.

A, Biological process

\begin{tabular}{llcr}
\hline Term & \multicolumn{1}{c}{ Name } & Count & P-value \\
\hline GO:0033036 & Macromolecule localization & 11 & $8.4 \times 10^{-6}$ \\
GO:0010033 & Response to organic substance & 10 & $2.1 \times 10^{-5}$ \\
GO:0002684 & Positive regulation of immune system process & 7 & $3.6 \times 10^{-5}$ \\
\hline
\end{tabular}

B, Cellular component

\begin{tabular}{llrr}
\hline Term & \multicolumn{1}{c}{ Name } & Count & P-value \\
\hline GO:0034358 & Plasma lipoprotein particle & 7 & $2.3 \times 10^{-12}$ \\
GO:1990777 & Lipoprotein particle & 7 & $2.3 \times 10^{-12}$ \\
GO:0032994 & Protein-lipid complex & 7 & $3.6 \times 10^{-12}$ \\
\hline
\end{tabular}

C, Molecular function

\begin{tabular}{llrr}
\hline Term & \multicolumn{1}{c}{ Name } & Count & P-value \\
\hline GO:0030169 & Low-density lipoprotein particle binding & 3 & $8.8 \times 10^{-5}$ \\
GO:0071813 & Lipoprotein particle binding & 3 & $1.6 \times 10^{-4}$ \\
GO:0071814 & Protein-lipid complex binding & 3 & $1.6 \times 10^{-4}$ \\
\hline
\end{tabular}

D, KEGG pathway

\begin{tabular}{|c|c|c|c|}
\hline Term & Name & Count & P-value \\
\hline ocu05323 & Rheumatoid arthritis & 6 & $1.3 \times 10^{-7}$ \\
\hline ocu05144 & Malaria & 4 & $5.0 \times 10^{-5}$ \\
\hline ocu05321 & Inflammatory bowel disease & 4 & $1.2 \times 10^{-4}$ \\
\hline
\end{tabular}

GO, Gene Ontology; KEGG, Kyoto Encyclopedia of Genes and Genomes.

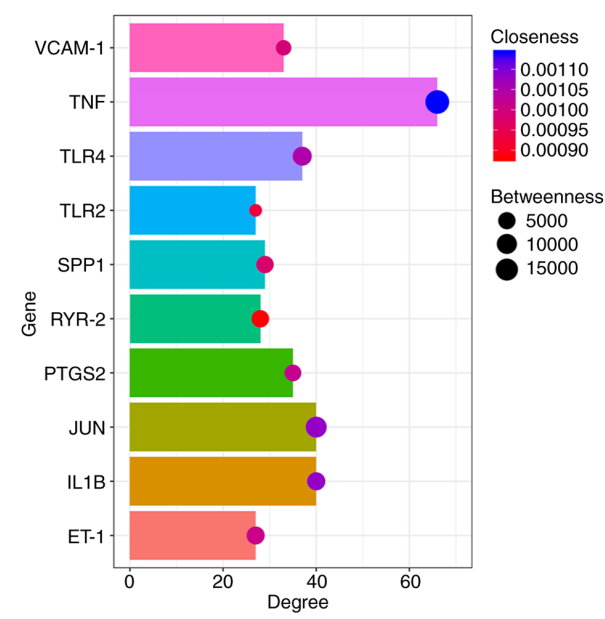

Figure 4. Betweenness, degree and closeness of hub genes. The top three hub genes with the highest degree, betweenness and closeness were TNF, JUN and IL-1 $\beta$. The X-axis represents the degree, the depth of the bubble color the closeness and the size of the bubbles the betweenness centrality. VCAM-1, vascular cell adhesion molecule 1; TLR4, Toll-like receptor 4; TLR2, Toll-like receptor 2; SPP1, secreted phosphoprotein 1; RYR-2, ryanodine receptor 2; PTGS2, prostaglandin-endoperoxide synthase 2; ET-1, ETS proto-oncogene 1 transcription factor. reticulum protein 1 was closely associated with the pathogenesis of PTE and a decreased endoplasmic reticulum stress response exhibited a crucial role in this process. Similarly, another study revealed that resveratrol was able to attenuate PTE via regulating the expression of metastasis-associated lung adenocarcinoma transcript 1 in lung tissues (21). In the present study, IL-1 $\beta$, JUN, TLR4, PTGS2, SPP1, TLR2 and ET-1 were identified to be the genes most closely associated with PTE in a microarray dataset from a rabbit model. To validate the significance of the aforementioned genes in the pathogenesis of PTE in humans, their mRNA expression levels were determined in patients with PTE and healthy controls. RT-qPCR analysis revealed that the expression of TLR4, TLR2, IL-1 $\beta$ and SPP1 was significantly upregulated in patients with PTE.

It has been reported that the TLR2/4-mediated NF- $\mathrm{B}$ signaling pathway is an important regulatory mechanism in coronary microembolization-induced myocardial injury (22). In addition, a previous study has highlighted the crucial role of TLR4 in the regulation of fat embolism syndrome via the TLR-JNK signaling pathway (23). 
Table VIII. Functional and pathway enrichment analysis of the top genes in module 2. The top three terms were selected based on the P-value when more than three enriched terms were identified in each category.

A, Biological process

\begin{tabular}{llrr}
\hline Term & \multicolumn{1}{c}{ Name } & Count & P-value \\
\hline GO:0045321 & Leukocyte activation & 9 & $6.7 \times 10^{-6}$ \\
GO:0006955 & Immune response & 10 & $1.2 \times 10^{-5}$ \\
GO:0001775 & Cell activation & 9 & $2.0 \times 10^{-5}$ \\
\hline
\end{tabular}

B, Cellular component

\begin{tabular}{llll}
\hline Term & \multicolumn{1}{c}{ Name } & Count & P-value \\
\hline GO:0016459 & Myosin complex & 5 & $7.0 \times 10^{-6}$ \\
GO:0009897 & External side of plasma membrane & 6 & $4.7 \times 10^{-5}$ \\
GO:0030016 & Myofibril & 5 & $1.6 \times 10^{-4}$ \\
\hline
\end{tabular}

C, Molecular functions

\begin{tabular}{llrr}
\hline Term & \multicolumn{1}{c}{ Name } & Count & P-value \\
\hline GO:0003774 & Motor activity & 4 & $9.6 \times 10^{-4}$ \\
GO:0005509 & Calcium ion binding & 5 & $1.3 \times 10^{-2}$ \\
GO:0008307 & Structural constituent of muscle & 2 & $2.7 \times 10^{-2}$ \\
\hline
\end{tabular}

D, KEGG pathway

\begin{tabular}{llll}
\hline Term & \multicolumn{1}{c}{ Name } & Count & P-value \\
\hline ocu04620 & Toll-like receptor signaling pathway & 6 & $7.0 \times 10^{-6}$ \\
ocu05140 & Toll-like receptor signaling pathway & 4 & $1.6 \times 10^{-3}$ \\
ocu05323 & Rheumatoid arthritis & 4 & $2.9 \times 10^{-3}$ \\
\hline
\end{tabular}

GO, Gene Ontology; KEGG, Kyoto Encyclopedia of Genes and Genomes.

In the present study, the expression of TLR4 and TLR2 was indicated to be markedly increased in patients with PTE compared with that in healthy controls. This finding combined with the bioinformatics analysis results suggested that TLR4 and TLR2 may serve as potential biomarkers for the diagnosis of PTE.

IL-1 $\beta$, also known as leukocytic pyrogen, leukocytic endogenous mediator, mononuclear cell factor or lymphocyte-activating factor, is a cytokine protein that is encoded by the IL-1 $\beta$ gene in humans (24). It has been previously reported that acute pulmonary embolism is associated with a significant release of IL-1 $\beta$ (25). SPP1 has been an important role in the regulation of cell-matrix interactions and endogenous ligands (26). In a previous study, the levels of SPP1 were measured in 119 patients with PTE and the results supported the potential roles of SPP1 in inflammatory and fibrotic processes in PTE. In the present study, the expression of IL-1 $\beta$ and SPP1 was significantly elevated in the serum of patients with PTE compared with that in the controls and both genes were identified to be closely associated with PTE. Therefore, it was hypothesized that IL-1 $\beta$ and SPP1 may be potential biomarkers for PTE. However, there is still a long way to go prior to the application of the current results in clinical practice. Since the incidence of PTE is significant, early diagnosis of the disease is of great importance. The results of the present study may provide researchers with additional knowledge in order to further investigate the mechanisms underlying PTE, with the aim that high-risk patients may be screened based on the expression of the PTE-related hub genes. In addition, these hub genes may be considered as potential therapeutic targets.

Similar to other bioinformatics-based studies, the present study has certain limitations. First, no in vivo experiments were performed to further confirm the role of hub genes in the pathogenesis of PTE. In addition, subtypes of PTE were not investigated in the present study. Therefore, further studies on the molecular mechanisms underlying the pathogenesis of various subtypes of PTE are urgently required (27).

Taken together, the present study demonstrated that the expression of TLR4, TLR2, IL-1 $\beta$ and SPP1 was increased in 
A

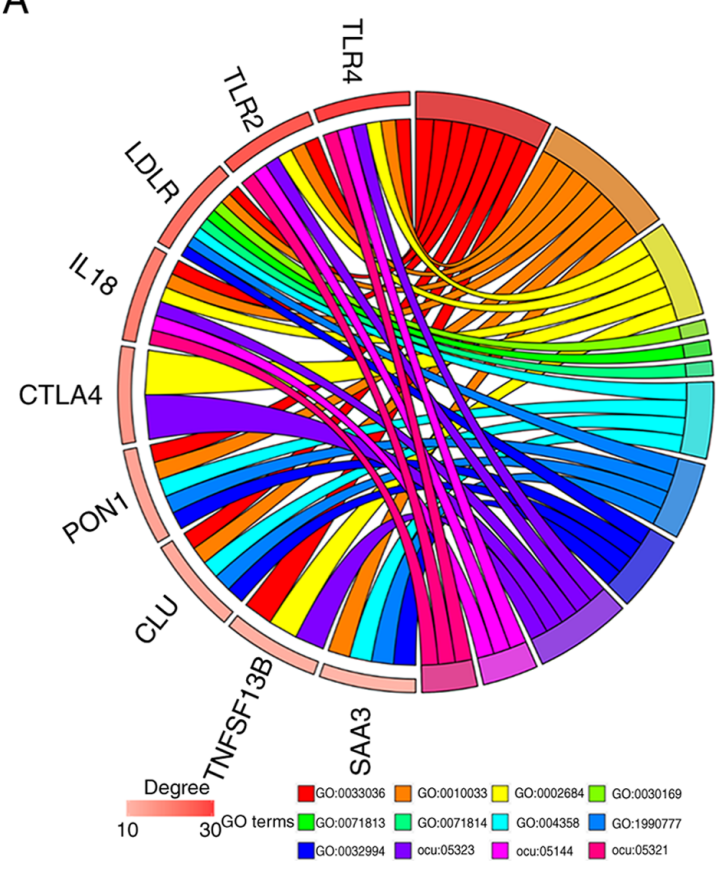

B

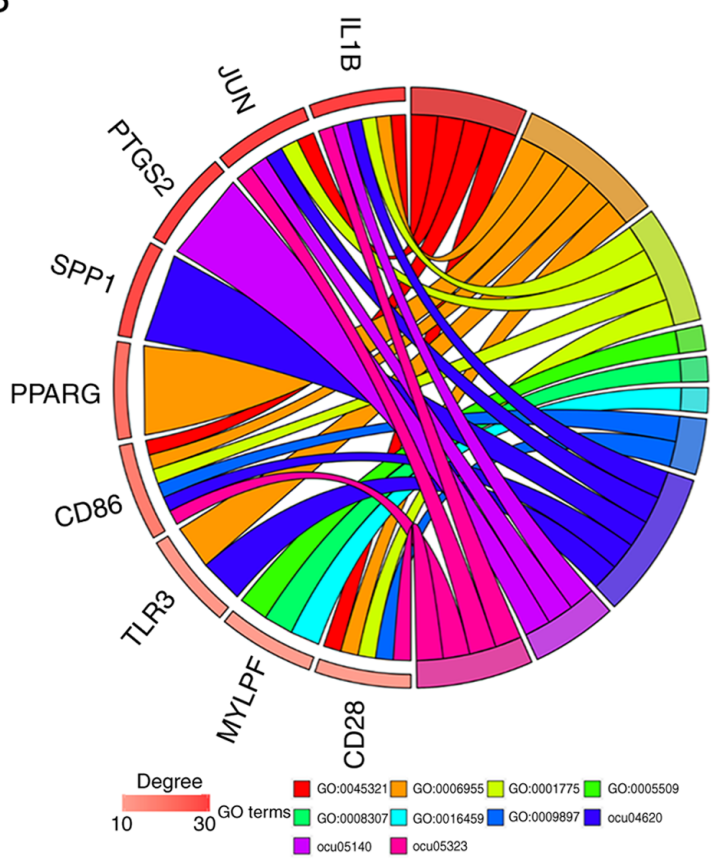

Figure 5. Top 10 enriched terms of modules 1 and 2. (A) The top 10 enriched terms of module 1. Biological process: GO:0033036, macromolecule localization; GO:0010033, response to organic substance; GO:0002684, positive regulation of immune system process. Molecular function: GO:0030169, low-density lipoprotein particle binding; GO:0071813, lipoprotein particle binding 3; GO:0071814, protein-lipid complex binding. Cellular component: GO:0034358, plasma lipoprotein particle; GO:1990777, lipoprotein particle; GO:0032994, protein-lipid complex. KEGG pathways: ocu05323, rheumatoid arthritis pathway; ocu05144, malaria pathway; ocu05321, inflammatory bowel disease pathway. (B) The top 10 enriched terms of module 2. Biological process: GO:0045321, leukocyte activation; GO:0006955, immune response; GO:0001775, cell activation. Molecular function: GO:0005509, calcium ion binding; GO:0008307, structural constituent of muscle Cellular component: GO:0016459, myosin complex; GO:0009897, external side of plasma membrane. KEGG pathways: ocu04620, Toll-like receptor signaling pathway; ocu05140, leishmaniasis; ocu05323, rheumatoid arthritis. GO, Gene Ontology; KEGG, Kyoto Encyclopedia of Genes and Genomes. TLR4, Toll-like receptor 4; TLR2, Toll-like receptor 2; LDLR, low density lipoprotein receptor; CTLA4, cytotoxic T-lymphocyte associated protein 4; PON1, paraoxonase 1; CLU, clusterin; TNFSF13B, TNF superfamily member 13b; SAA3, serum amyloid A3, pseudogene; PTGS2, prostaglandin-endoperoxide synthase 2; SPP1, secreted phosphoprotein 1; PPARG, peroxisome proliferator activated receptor gamma; CD86, CD86 molecule; TLR3, Toll-like receptor 3; MYLPF, myosin light chain.

A

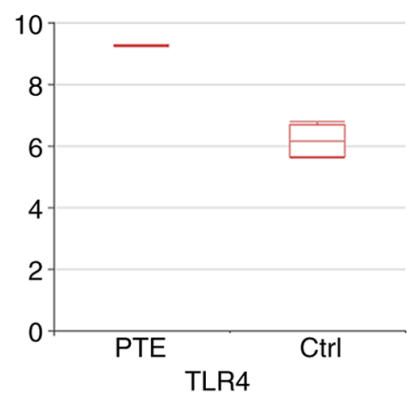

$\mathrm{E}$
B

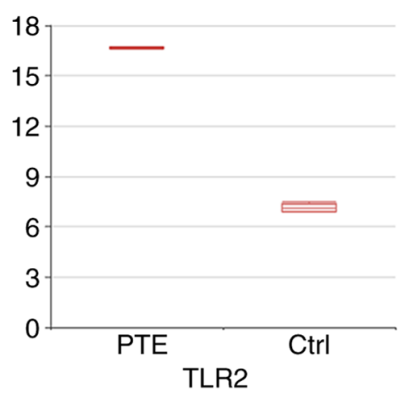

$\mathrm{F}$
C

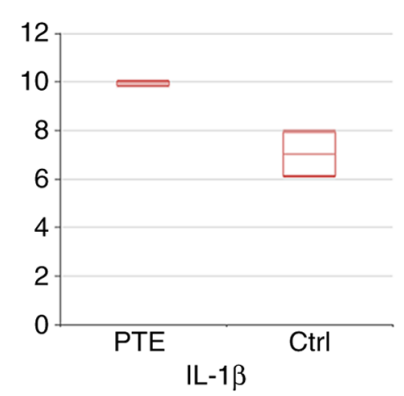

D

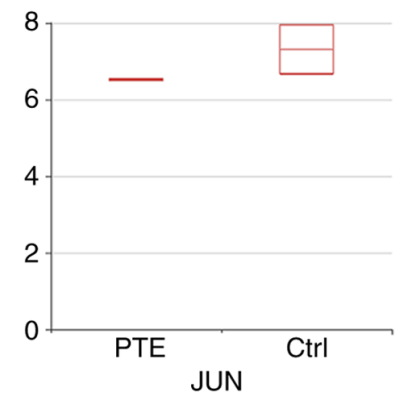

G
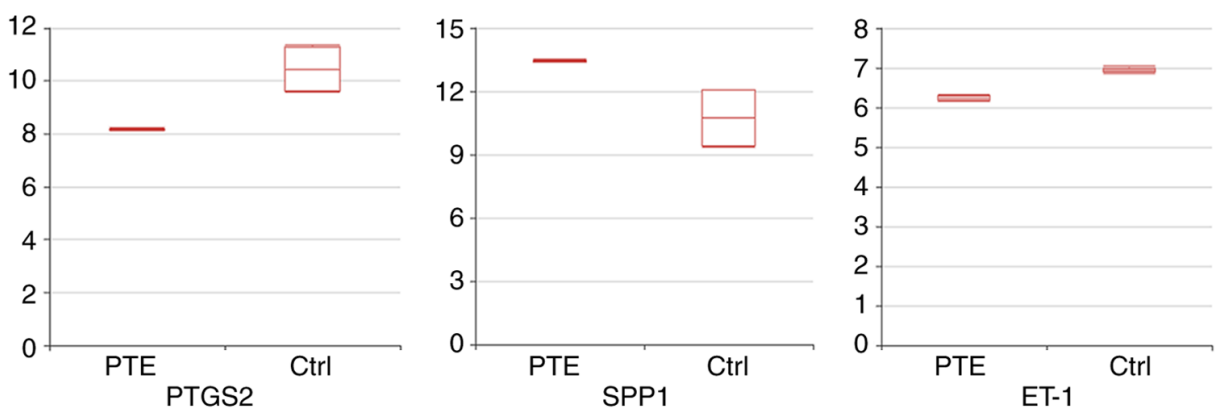

Figure 6. Expression levels of the indicated markers. Boxplots indicating the expression levels of (A) TLR4 (B) TLR2, (C) IL-1 $\beta$, (D) JUN, (E) PTGS2, (F) SPP1 and (G) ET-1 (mean \pm SD) were visualized using the Morpheus online tool. The Y-axis indicates the normalized expression value of the genes obtained from the series matrix files of the GSE84738 dataset. TLR, Toll-like receptor; PTGS2, prostaglandin-endoperoxide synthase 2; SPP1, osteopontin; ET-1, endothelin-1; Ctrl, control; PTE, pulmonary thromboembolism. 
A

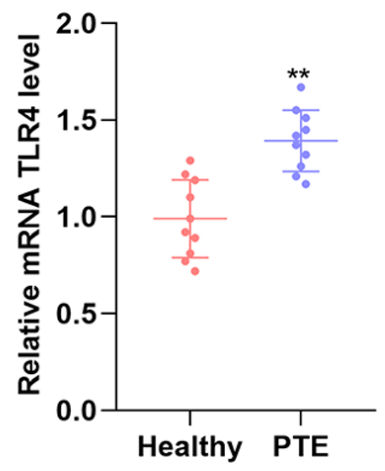

E

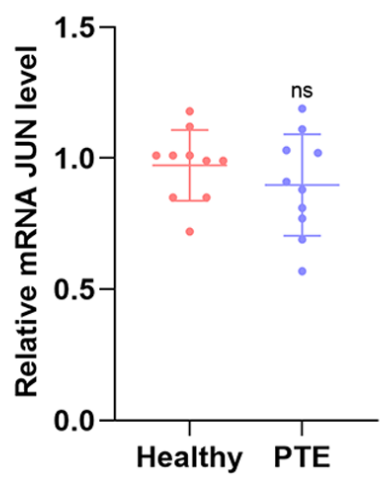

B

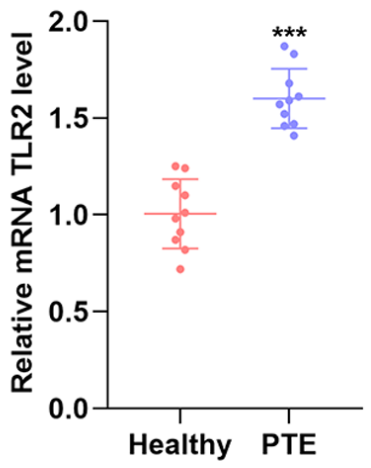

$\mathbf{F}$

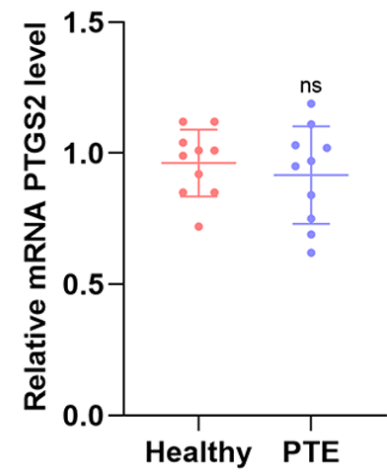

C

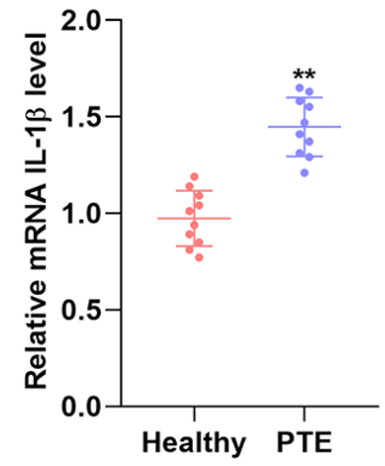

G

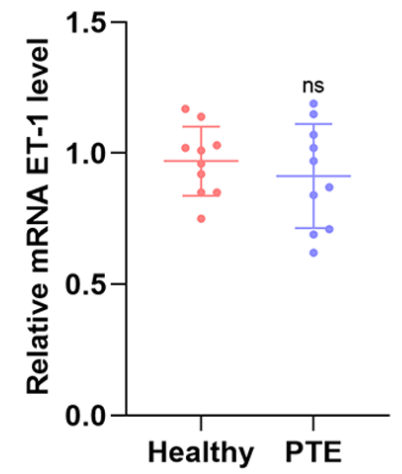

Figure 7. mRNA expression levels of TLR4, TLR2, IL-1 $\beta$ and SPP1. The mRNA expression levels of (A) TLR4, (B) TLR2, (C) IL-1 $\beta$, (D) SPP1, (E) JUN, (F) PTGS2 and (G) ET-1 in patients with PTE and healthy controls ( $\mathrm{n}=10$, per group) were determined by reverse transcription-quantitative PCR. Values are expressed as the mean \pm standard deviation from three independent experiments. ${ }^{* *} \mathrm{P}<0.01$ and ${ }^{* * *} \mathrm{P}<0.001$ vs. healthy group. The $\mathrm{Y}$-axis represents the relative mRNA expression (target gene/ $\beta$-actin). TLR, Toll-like receptor; SPP1, osteopontin; PTE, pulmonary thromboembolism; PTGS-2, prostaglandin-endoperoxide synthase 2; ET-1, endothelin-1.

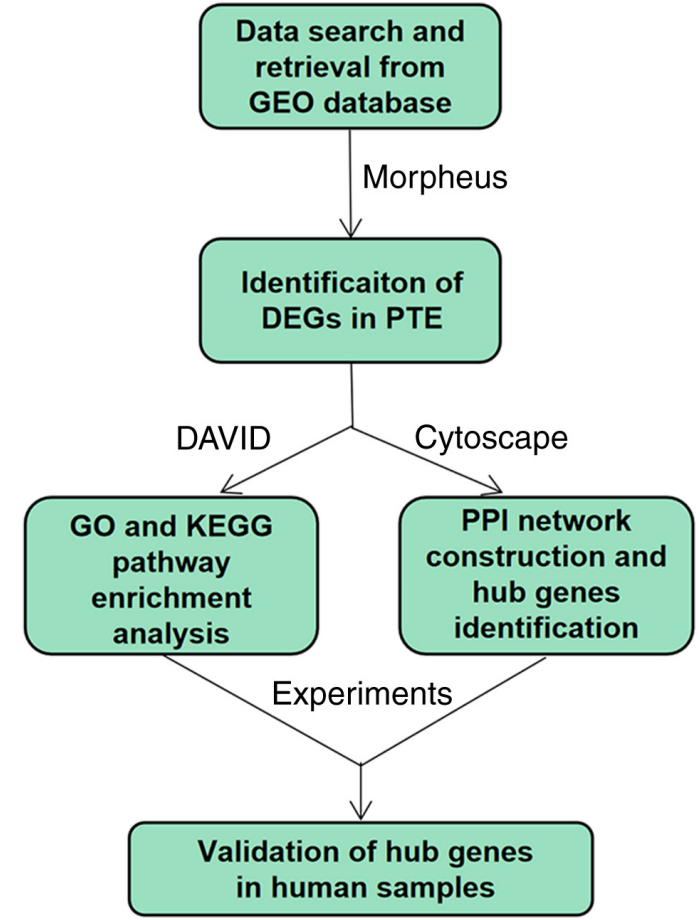

Figure 8. Schema of the study. GEO, Gene Expression Omnibus; DEGs, differentially expressed genes; PTE, pulmonary thromboembolism; GO, Gene Ontology; KEGG, Kyoto Encyclopedia of Genes and Genomes; PPI, protein-protein interaction. patients with PTE using both bioinformatics analyses and validation experiments, thus providing novel insight into potential biomarkers for the increasing risk of PTE.

\section{Acknowledgements}

Not applicable.

\section{Funding}

The present study was supported by the Construction of Key Medical Specialties in Shanghai (grant no. ZK2019-B05) and the Clinical Characteristics of Health System in Pudong New Area, Shanghai (grant no. PWYts2018-02).

\section{Availability of data and materials}

The datasets used and/or analyzed during the current study are available from the corresponding author on reasonable request.

\section{Authors' contributions}

PC and JZ designed the study. YL and JD performed the experiments. QL collected the data and performed the statistical analysis. YL and JD prepared the manuscript. YL and 
PC check and confirm the authenticity of the data. All authors read and approved the final manuscript.

\section{Ethics approval and consent to participate}

The present study was approved by the Committees of Clinical Ethics of Shanghai University of Medicine and Health Sciences Affiliated Zhoupu Hospital (Shanghai, China; approval no. 2019-042-84). Written informed consent was obtained from all patients.

\section{Patient consent for publication}

Not applicable.

\section{Competing interests}

The authors declare that they have no competing interests.

\section{References}

1. Brekelmans MP, Ageno W, Beenen LF, Brenner B, Buller HR, Chen CZ, Cohen AT, Grosso MA, Meyer G, Raskob G, et al: Recurrent venous thromboembolism in patients with pulmonary embolism and right ventricular dysfunction: A post-hoc analysis of the Hokusai-VTE study. Lancet Haematol 3: e437-e445, 2016.

2. Fang MC, Fan D, Sung SH, Witt DM, Schmelzer JR, Williams MS, Yale SH, Baumgartner C and Go AS: Treatment and outcomes of acute pulmonary embolism and deep venous thrombosis: The CVRN VTE study. Am J Med 132: 1450-1457.e1, 2019.

3. Coquoz N, Weilenmann D, Stolz D, Popov V, Azzola A, Fellrath JM, Stricker H, Pagnamenta A, Ott S, Ulrich S, et al: Multicentre observational screening survey for the detection of CTEPH following pulmonary embolism. Eur Respir J 51: 1702505, 2018.

4. Jiménez D, Bikdeli B, Quezada A, Muriel A, Lobo JL, de Miguel-Diez J, Jara-Palomares L, Ruiz-Artacho P, Yusen RD and Monreal M; RIETE investigators: Hospital volume and outcomes for acute pulmonary embolism: Multinational population based cohort study. BMJ 366: 14416, 2019.

5. Becattini C, Giustozzi M, Cerdà P, Cimini LA, Riera-Mestre A and Agnelli G: Risk of recurrent venous thromboembolism after acute pulmonary embolism: Role of residual pulmonary obstruction and persistent right ventricular dysfunction. A meta-analysis. J Thromb Haemost 17: 1217-1228, 2019.

6. Xiong Y, Mi BB, Liu MF, Xue H, Wu QP and Liu GH: Bioinformatics analysis and identification of genes and molecular pathways involved in synovial inflammation in rheumatoid arthritis. Med Sci Monit 25: 2246-2256, 2019.

7. Yu T, You X, Zhou H, Kang A, He W, Li Z, Li B, Xia J, Zhu H, Zhao Y, et al: p53 plays a central role in the development of osteoporosis. Aging (Albany NY) 12: 10473-10487, 2020.

8. Yu T, Xiong Y, Luu S, You X, Li B, Xia J, Zhu H, Zhao Y, Zhou H, Yu G and Yang Y: The shared KEGG pathways between icariin-targeted genes and osteoporosis. Aging (Albany NY) 12: 8191-8201, 2020 .

9. Xiong Y, Cao F, Chen L, Yan C, Zhou W, Chen Y, Endo Y, Leng X, Mi B and Liu G: Identification of key microRNAs and target genes for the diagnosis of bone nonunion. Mol Med Rep 21: 1921-1933, 2020.

10. Yu T, Wang Z, You X, Zhou H, He W, Li B, Xia J, Zhu H, Zhao Y, Yu G, et al: Resveratrol promotes osteogenesis and alleviates osteoporosis by inhibiting p53. Aging (Albany NY) 12: 10359-10369, 2020.

11. Desch KC, Ozel AB, Halvorsen M, Jacobi PM, Golden K, Underwood M, Germain M, Tregouet DA, Reitsma PH, Kearon $\mathrm{C}$, et al: Whole-exome sequencing identifies rare variants in STAB2 associated with venous thromboembolic disease. Blood 136: 533-541, 2020.
12. Veninga A, De Simone I, Heemskerk JWM, Cate HT and van der Meijden PEJ: Clonal hematopoietic mutations linked to platelet traits and the risk of thrombosis or bleeding. Haematologica 105: 2020-2031, 2020

13. Mi B, Liu G, Zhou W, Lv H, Zha K, Liu Y, Wu Q and Liu J: Bioinformatics analysis of fibroblasts exposed to TGF- $\beta$ at the early proliferation phase of wound repair. Mol Med Rep 16: 8146-8154, 2017.

14. Huang da W, Sherman BT and Lempicki RA: Systematic and integrative analysis of large gene lists using DAVID bioinformatics resources. Nat Protoc 4: 44-57, 2009.

15. von Mering C, Jensen LJ, Snel B, Hooper SD, Krupp M, Foglierini M, Jouffre N, Huynen MA and Bork P: STRING: Known and predicted protein-protein associations, integrated and transferred across organisms. Nucleic Acids Res 33 (Database Issue): D433-D437, 2005.

16. Shannon P, Markiel A, Ozier O, Baliga NS, Wang JT, Ramage D, Amin N, Schwikowski B and Ideker T: Cytoscape: A software environment for integrated models of biomolecular interaction networks. Genome Res 13: 2498-2504, 2003.

17. Bader GD and Hogue CW: An automated method for finding molecular complexes in large protein interaction networks. BMC Bioinformatics 4: 2, 2003.

18. Livak KJ and Schmittgen TD: Analysis of relative gene expression data using real-time quantitative PCR and the 2(-Delta Delta C(T)) method. Methods 25: 402-408, 2001.

19. Essien EO, Rali P and Mathai SC: Pulmonary embolism. Med Clin North Am 103: 549-564, 2019.

20. Liu TW, Liu F and Kang J: Let-7b-5p is involved in the response of endoplasmic reticulum stress in acute pulmonary embolism through upregulating the expression of stress-associated endoplasmic reticulum protein 1. IUBMB Life 72: 1725-1736, 2020.

21. Yang K, Li W, Duan W, Jiang Y, Huang N, Li Y, Ren B and Sun J: Resveratrol attenuates pulmonary embolism associated cardiac injury by suppressing activation of the inflammasome via the MALAT1-miR-22-3p signaling pathway. Int J Mol Med 44: 2311-2320, 2019

22. Su Q, Lv X, Sun Y, Ye Z, Kong B and Qin Z: Role of TLR4/MyD88/NF- $\kappa$ B signaling pathway in coronary microembolization-induced myocardial injury prevented and treated with nicorandil. Biomed Pharmacother 106: 776-784, 2018.

23. Xiong L, Sun L, Liu S, Zhu X, Teng Z and Yan J: The protective roles of urinary trypsin inhibitor in brain injury following fat embolism syndrome in a rat model. Cell Transplant 28: 704-712, 2019.

24. Licata G, Tuttolomondo A, Di Raimondo D, Corrao S, Di Sciacca R and Pinto A: Immuno-inflammatory activation in acute cardio-embolic strokes in comparison with other subtypes of ischaemic stroke. Thromb Haemost 101: 929-937, 2009.

25. Sun TW, Zhang JY, Li L and Wang LX: Effect atorvastatin on serum tumor necrosis factor alpha and interleukin-1 $\beta$ following acute pulmonary embolism. Exp Lung Res 37: 78-81, 2011.

26. von Zur Mühlen C, Koeck T, Schiffer E, Sackmann C, Zürbig P, Hilgendorf I, Reinöhl J, Rivera J, Zirlik A, Hehrlein C, et al: Urine proteome analysis as a discovery tool in patients with deep vein thrombosis and pulmonary embolism. Proteomics Clin Appl 10: 574-584, 2016.

27. Kölmel S, Hobohm L, Käberich A, Krieg VJ, Bochenek ML, Wenzel P, Wiedenroth CB, Liebetrau C, Hasenfuß G, Mayer E, et al: Potential involvement of osteopontin in inflammatory and fibrotic processes in pulmonary embolism and chronic thromboembolic pulmonary hypertension. Thromb Haemost 119: 1332-1346, 2019.

This work is licensed under a Creative Commons Attribution-NonCommercial-NoDerivatives 4.0 International (CC BY-NC-ND 4.0) License. 